\title{
Regulation of Chondrocyte Differentiation by
}

\section{Changing Intercellular Distances Using Collagen}

\section{Type II Microfibers}

Jinyu Li ${ }^{1}$, Naoko Sasaki ${ }^{2}$, Keiji Itaka ${ }^{3}$, Margo Terpstra ${ }^{4}$, Riccardo Levato ${ }^{5}$, and Michiya Matsusakil,2*

${ }^{1}$ Department of Applied Chemistry, Graduate School of Engineering, Osaka University, Osaka 565-0871, Japan

${ }^{2}$ Joint Research Laboratory (TOPPAN) for Advanced Cell Regulatory Chemistry, Graduate

School of Engineering, Osaka University, Osaka 565-0871, Japan

${ }^{3}$ Department of Biofunction Research, Institute of Biomaterials and Bioengineering, Tokyo Medical and Dental University (TMDU), Tokyo 101-0062, Japan

${ }^{4}$ Department of Orthopaedics, University Medical Center Utrecht, Utrecht University, Utrecht 3584CX, The Netherlands

${ }^{5}$ Department of Clinical Sciences, Faculty of Veterinary Medicine, Utrecht University, Utrecht, 3584CM, The Netherlands

* Corresponding authors. Phone: +81-6-6879-7356; Fax: +81-6-6879-7359; Email: mmatsus@chem.eng.osaka-u.ac.jp

The supplementary information contains the following sections:

Figures S1-S7. 


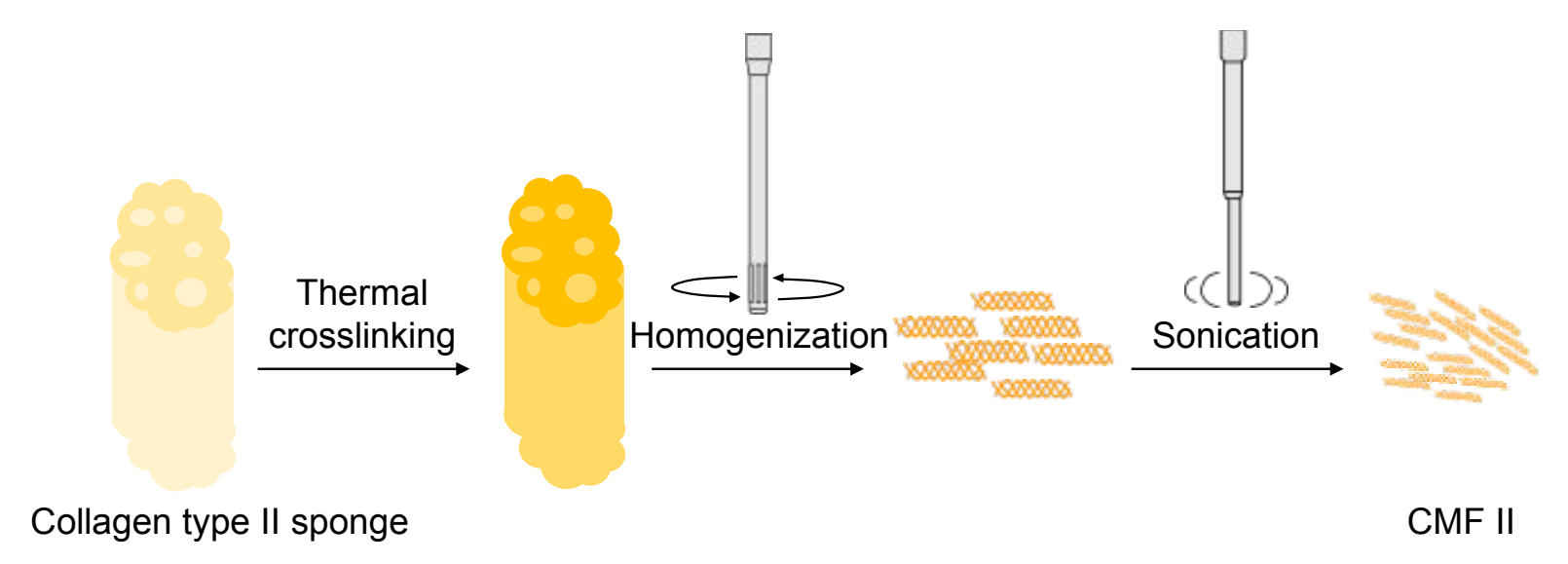

Figure S1. The workflows of sizing down the collagen type II sponge into CMF II through mechanical processes. The color of the collagen type II sponge was slightly changed after dehydration due to the thermal crosslinking.

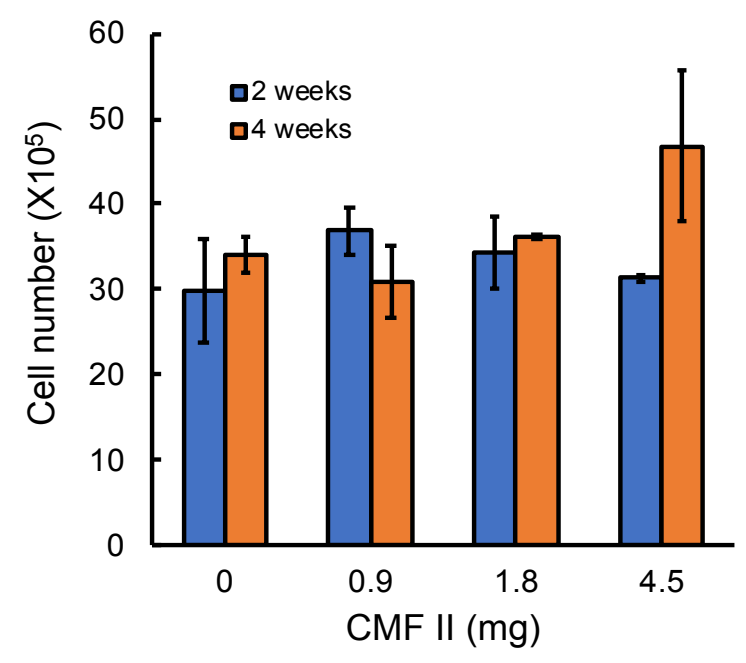

Figure S2. Time and CMF II independent cell growth of constructed 3D cartilage tissues. Cell number is determined by DNA content measured by Nanodrop 1000. DNA of each sample was isolated and purified ahead guided by DNeasy Blood \& Tissue Kits. $n=3$. 


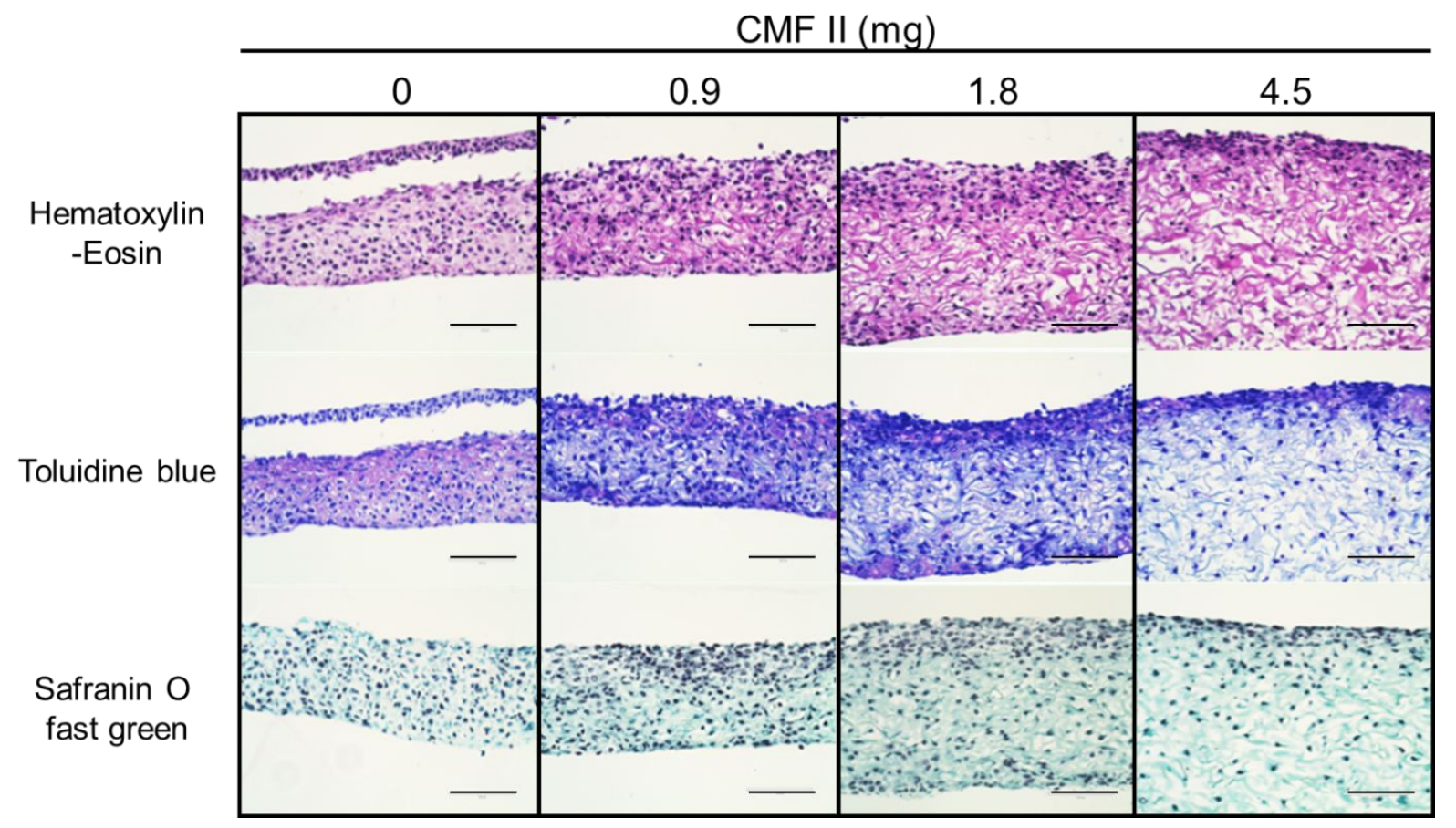

Figure S3. Immunohistochemistry outcomes of constructed cartilage tissues after two weeks differentiation in medium A. Abundant matrix (pink observed in Hematoxylin-Eosin stain) was presented in constructed cartilage tissues. glycosaminoglycan (purple observed in toluidine blue stain) was expressed more strongly in fewer CMF II containing cartilage tissues. Proteoglycan (red observed in safranin O fast green stain) was hardly detected in all cartilage tissues. Scale bar: $100 \mu \mathrm{m}$. 


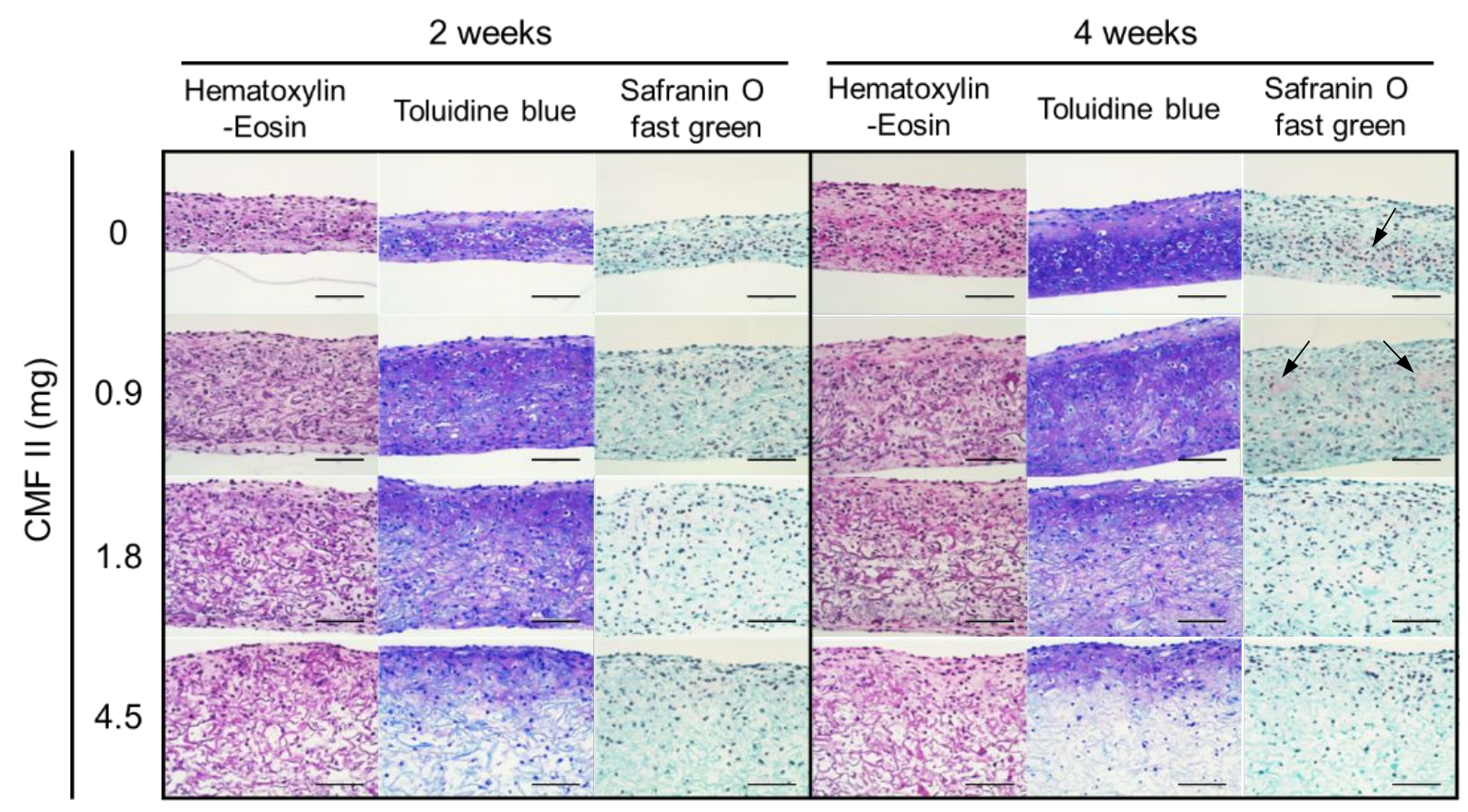

Figure S4. Histologic evaluation of medium B differentiated constructed 3D cartilage tissues. The cartilage specific expression of glycosaminoglycan (purple) and slight proteoglycan (red) indicated by black arrows were detected in the above images. Scale bar: $100 \mu \mathrm{m}$. 


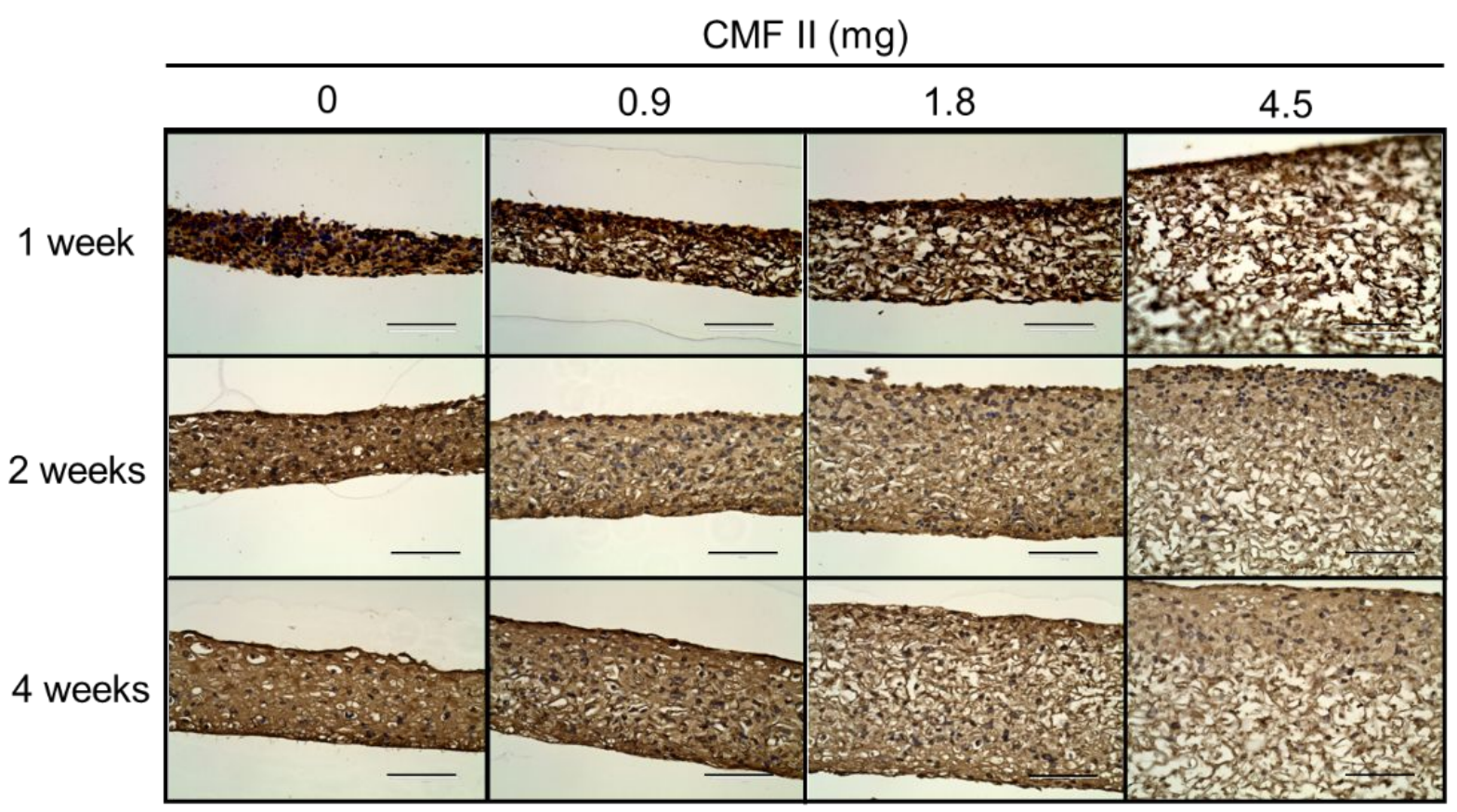

Figure S5. Histology analysis of Col II immunohistochemistry stained cartilage tissue sections. Cartilaginous phenotype was confirmed by positive stained type II collagen (brown). Scale bar: $100 \mu \mathrm{m}$. 


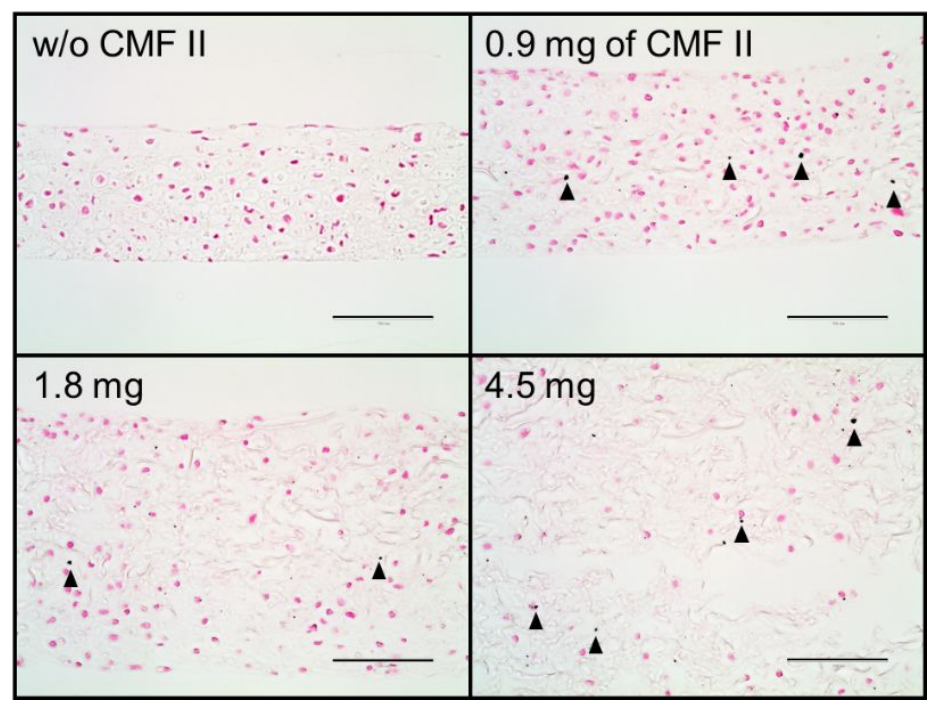

Figure S6. Histological analysis of Von Kossa stained cartilage tissues. The cartilage tissues presented above were differentiated for 4 weeks. Black spots indicated by arrowheads hinted the calcium deposits. Scale bar: $100 \mu \mathrm{m}$.

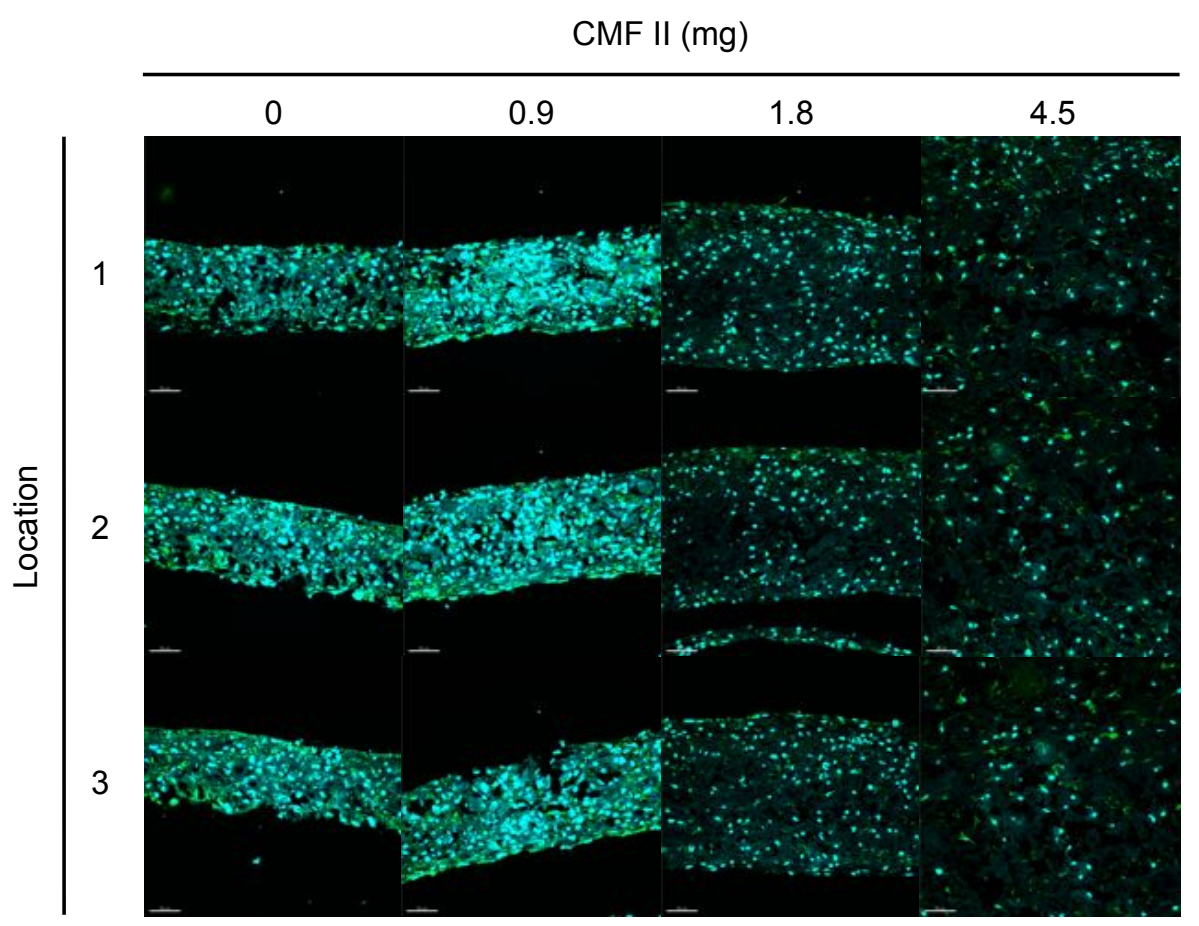

Figure S7. N-Cadherin expression varied in constructed cartilage tissues with different intercellular distances. For each sample, three locations were selected for fluorescence signaling quantification. N-Cadherin is indicated in green by Alexa Fluor ${ }^{\circledR} 488$ and nuclei is indicated in blue by DAPI. Scale bar: $50 \mu \mathrm{m}$. 